\title{
Albumin Infusion in Patients with Cirrhosis: Time for POCUS-Enhanced Physical Examination
}

\author{
Abhilash Koratala $^{a}$ Claudio Ronco $^{b, c}$ Amir Kazory $^{d}$

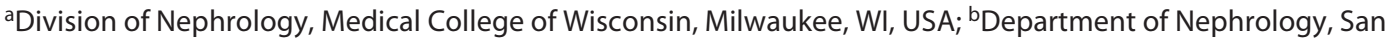 \\ Bortolo Hospital and International Renal Research Institute of Vicenza (IRRIV), Vicenza, Italy; 'Department of \\ Medicine, University of Padova, Padova, Italy; ${ }^{d}$ Division of Nephrology, Hypertension and Renal Transplantation, \\ University of Florida, Gainesville, FL, USA
}

\section{Keywords}

Point-of-care ultrasound - Physical examination - Cirrhosis .

Albumin $\cdot$ Nephrology $\cdot$ Hepatorenal syndrome

\begin{abstract}
Objective assessment of fluid status is of utmost significance in the management of patients with complex disorders involving hemodynamics and multi-organ crosstalk such as cardiorenal or hepatorenal syndrome. The role of volume expansion using intravenous albumin in the setting of hepatorenal syndrome has been an everlasting debate among clinicians. With the accumulating evidence on the deleterious consequences of iatrogenic fluid overload, empiric albumin administration in these patients has been the focus of much attention, and the findings of recent studies suggest a higher incidence of pulmonary complications with albumin. Poor sensitivity of conventional physical examination has led to an interest in the utility of novel noninvasive bedside tools such as point-of-care ultrasonography (POCUS) to evaluate hemodynamics more precisely. Once confined to specialties such as obstetrics and emergency medicine, the scope of diagnostic POCUS is rapidly expanding in other fields including internal medicine and nephrology. Herein, we offer our perspective on the emerging role of POCUS for
\end{abstract}

objective evaluation of patients with suspected hepatorenal physiology based on our experience. We propose that future clinical trials consider incorporating this strategy and explore the impact of POCUS-guided therapy on the outcomes.

(c) 2021 The Author(s).

Published by S. Karger AG, Basel

Accurate assessment of fluid status is of paramount importance in guiding the management of any condition that involves hemodynamic aberrancy and subsequent organ dysfunction. Whether to administer intravenous fluids or use diuretics ("to fill or to drain") is a common clinical dilemma faced by clinicians providing care for patients with complex hemodynamic disorders. In certain circumstances, neither of these strategies would be appropriate; protocol-based or empiric therapies based on clinical gestalt may result in unintended adverse consequences. Cirrhosis and/or hepatorenal syndrome (HRS) is one such example where the role of volume expansion using intravenous albumin, a commonly chosen strategy, has been frequently debated. In a recent randomized controlled trial including patients with decompensated cirrhosis, repeated daily infusion of intravenous albumin targeting a serum level $>3 \mathrm{~g} / \mathrm{dL}$ did not improve karger@karger.com www.karger.com/crm

Karger $\stackrel{\text { ' }}{5}$
(C) 2021 The Author(s)

Published by S. Karger AG, Basel

This is an Open Access article licensed under the Creative Commons Attribution-NonCommercial-4.0 International License (CC BY-NC) (http://www.karger.com/Services/OpenAccessLicense), applicable to the online version of the article only. Usage and distribution for commercial purposes requires written permission.
Correspondence to:

Abhilash Koratala, akoratala@mcw.edu 


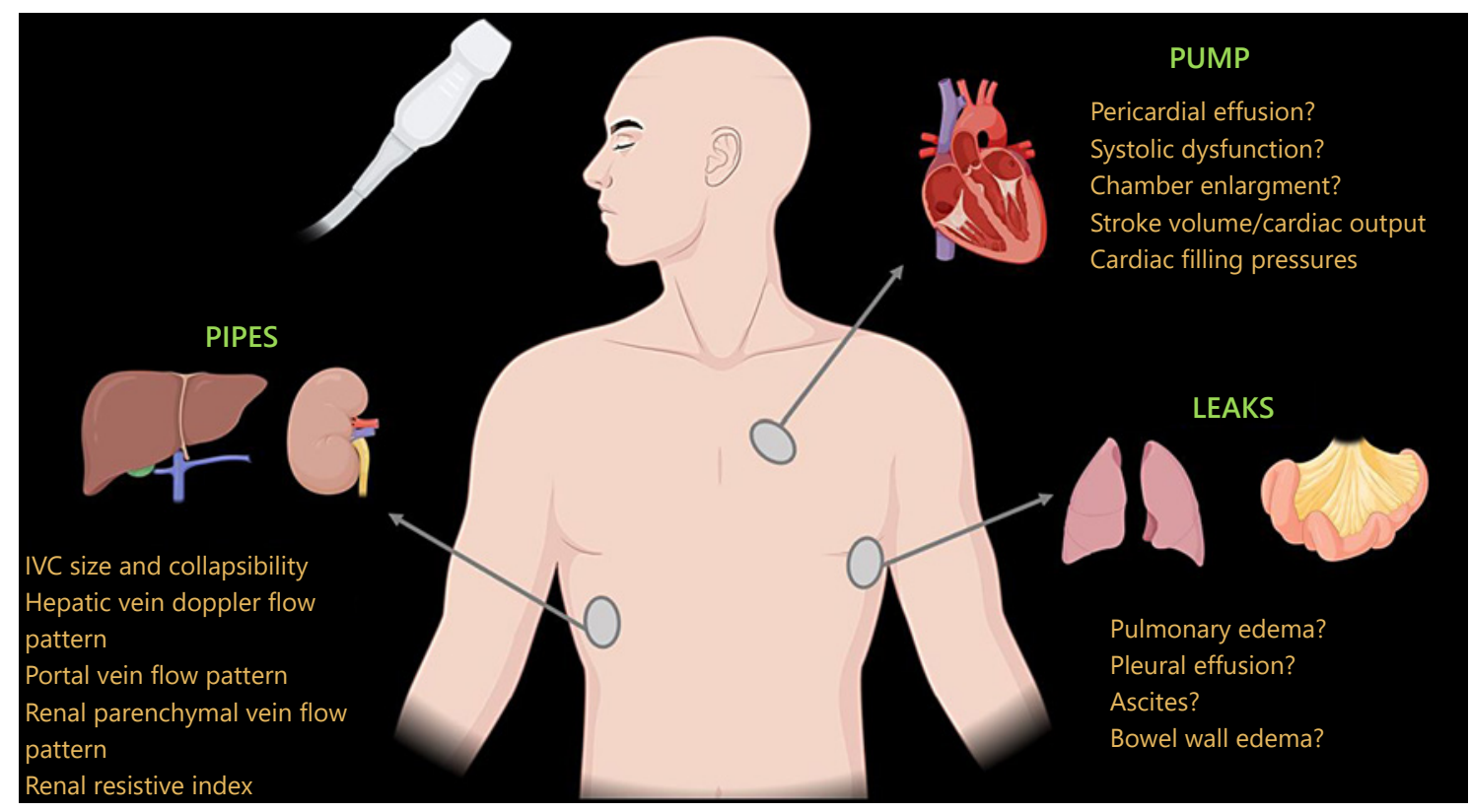

Fig. 1. Illustration of the pump-pipes-leaks model of fluid volume status assessment showing common focused questions answered/parameters assessed by clinician-performed ultrasound at the bedside. Circles on the body are representative sonographic windows; alternates exist. Illustrations made using Biorender. IVC, inferior vena cava.

renal function compared to standard care, while it did increase the incidence of adverse events [1]. Of note, patients who received albumin were more likely to develop pulmonary edema and fluid overload. Similar findings were observed in a study evaluating the efficacy of albumin infusion in patients with cirrhosis and sepsis [2]. Moreover, in the CONFIRM trial investigating the efficacy of terlipressin in HRS, the incidence of respiratory failure was significantly higher in the treatment arm [3]. Notably, albumin was coadministered in $83 \%$ of these patients, which could have had a synergistic effect in causing pulmonary edema together with terlipressin-induced increased afterload.

These observations call for further discussion as the relationship between fluid overload and mortality is being increasingly recognized [4]. Furthermore, a negative mean daily fluid balance was shown to be associated with improved clinical outcomes and increased dialysis-free days in critically ill patients with acute kidney injury (AKI) [5]. While clinicians frequently rely on the physical examination findings and weight to assess fluid status, sensitivity of these parameters has been questioned and point-of-care ultrasonography (POCUS) has emerged as a valuable adjunct over the past few years. For instance, in a large cohort of critically ill patients, the sensitivity of auscultation was only $52 \%$ to detect pulmonary edema diagnosed by lung ultrasound [6]. Similarly, in a study of 53 patients with "clinical" diagnosis of HRS type 1, 64\% were found to have alternative diagnoses such as intravascular fluid overload and intra-abdominal hypertension based on inferior vena cava (IVC) ultrasound findings [7]. In some patients, particularly those with heart failure, fluid overload could be due to volume redistribution rather than absolute fluid gain; weight changes do not necessarily reflect congestion [8]. This is of significance as cardiac inotropic and chronotropic dysfunction is thought to play a key role in the circulatory derangement observed in HRS [9].

Bedside sonographic evaluation of the pump, pipes, and the leaks (Fig. 1) provides valuable insights into hemodynamics in such patients and could potentially avoid iatrogenic fluid overload [10]. Pump indicates focused cardiac ultrasound, pipes refer to ultrasound of the IVC and abdominal veins to detect congestion, and leaks denote lung and abdominal ultrasound to detect extravascular lung water, ascites, and gut edema. This multi-parametric approach addresses the shortcomings and technical difficulties associated with individual sonographic applications. For example, portal vein Doppler flow can be pulsatile in cirrhosis due to portal hypertension, with- 


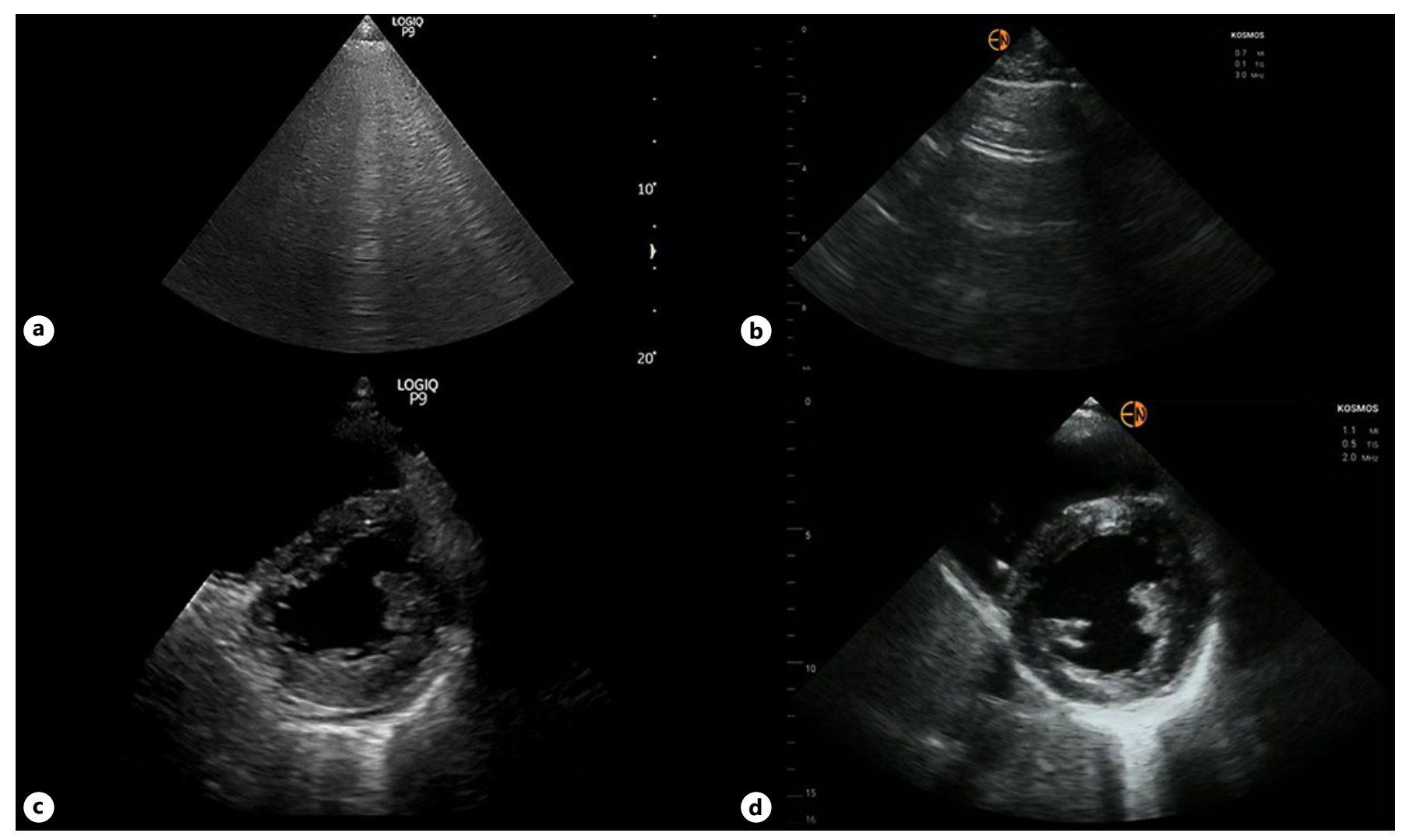

Fig. 2. [Upper panel] Lung ultrasound demonstrating vertical artifacts (B-lines) prior to diuretic therapy, suggestive of congestion (right upper lateral zone shown) (a) and normal horizontal artifacts (A-lines) after diuretics (b). [Lower panel] Parasternal short axis view of the heart demonstrating interventricular septal flattening leading to "D-shaped" left ventricle prior to diuretic therapy (c); normalized interventricular septum with left ventricle assuming round shape after diuretics (d). ${ }^{*}$ Different ultrasound machine was used for follow-up exam. out an elevation in right atrial pressure [11]. Conversely, IVC diameter can be falsely low in patients with increased intra-abdominal pressure (e.g., tense ascites) despite intravascular volume excess. In addition, some sonographic windows may be difficult than others in a given patient due to a variety of reasons such as body habitus, bowel gas, and altered echotexture of the cirrhotic liver.

HRS is essentially a diagnosis of exclusion, but the socalled hepatorenal pathophysiology overlaps with several other causes of AKI seen in patients with liver disease. It generally refers to arterial vasodilatation induced by portal hypertension and pooling of blood in the splanchnic circulation leading to renal hypoperfusion. Consequent activation of the renin-angiotensin-aldosterone system (RAAS) and neurohormonal adaptations lead to intense renal vasoconstriction. In addition, arterial underfilling leads to activation of the baroreceptor reflex and sympathetic nervous system, resulting in a hyperdynamic state with increased heart rate, stroke volume, and cardiac output. Renal sodium and water retention due to RAAS activation expands the plasma volume, which increases the cardiac preload leading to further upsurge in cardiac output. This subsequently increases the portal flow and pressure, thus perpetuating the vicious cycle [12]. Increased preload also elevates the cardiac filling pressures, making the patient susceptible to developing pulmonary edema with intravenous fluid therapy. Some patients with liver disease progress to a state of cirrhotic cardiomyopathy with decreased cardiac output, complicating the clinical picture. The cardiac component of hepatorenal physiology has been increasingly recognized and is of such clinical significance that we have previously proposed the term hepatocardiorenal syndrome [9]. Further complicating the matter, a subset of these patients present with true volume depletion. Multi-organ POCUS allows rapid and objective assessment of the cardiac pump function, 


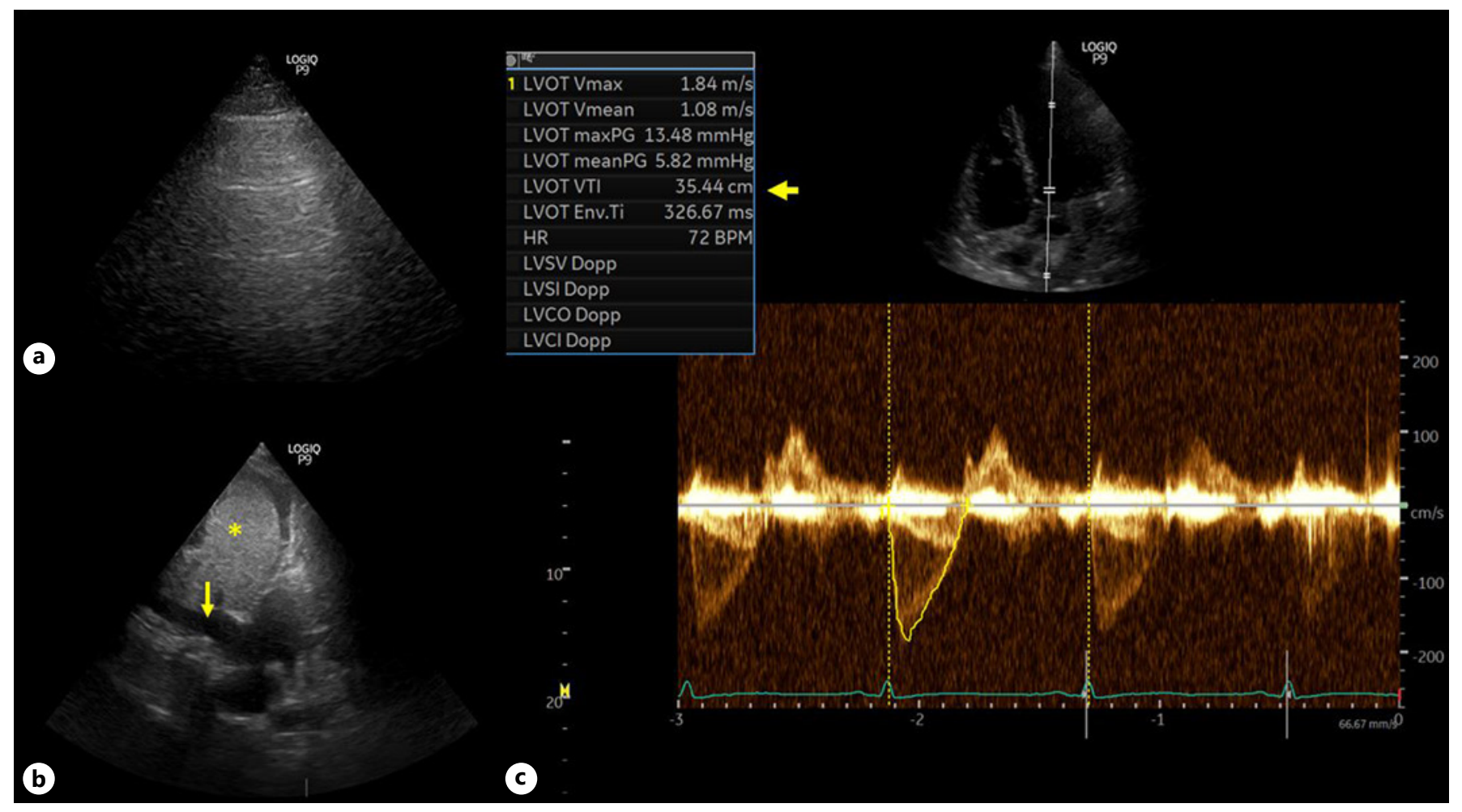

Fig. 3. Lung ultrasound demonstrating A-lines (right upper zone shown) (a); IVC (arrow) ultrasound showing a relatively small vessel $(\sim 1.7 \mathrm{~cm})$. Note the hyperechoic cirrhotic liver (asterisk) (b); left ventricular outflow tract pulsed wave Doppler demonstrating supra-normal VTI of $35.5 \mathrm{~cm}$ (c). VTI is a measure of stroke volume, which is normally $\sim 18-22 \mathrm{~cm}$. IVC, inferior vena cava; VTI, velocity time integral. including stroke volume, lung water, and venous congestion at bedside, thereby guiding therapy. Sonographic markers of cardiac filling pressures and renal vasoconstriction such as resistive index could further aid in the diagnostic process. Although portending certain limitations, high renal resistive index may point toward HRS as opposed to AKI from volume depletion when interpreted in the appropriate clinical context $[13,14]$.

The following real-world examples would help further clarify the aforementioned concepts related to the role of POCUS in guiding the management of patients with cirrhosis and hepatocardiorenal physiology. Case 1: a young man with liver cirrhosis was admitted for incarcerated inguinal hernia repair. Serum creatinine worsened to 2.2 $\mathrm{mg} / \mathrm{dL}$ two days after the surgery from a baseline of 0.6 $\mathrm{mg} / \mathrm{dL}$. There was associated hyperbilirubinemia and elevated liver enzymes. He was started on daily intravenous albumin infusions for presumed volume depletion and/ or HRS, but renal function continued to decline. POCUS evaluation revealed a diffuse B-line pattern in the lung, suggestive of pulmonary edema. In addition, there was interventricular septal flattening during diastole on focused cardiac ultrasound, suggestive of intravascular volume overload. Interestingly, he had only trace pedal ede$\mathrm{ma}$, and lung auscultation was documented as clear by 2 different physicians. Based on the POCUS findings, the management changed; albumin administration was stopped, and intravenous diuretics were initiated. Renal function improved gradually. Follow-up POCUS revealed resolution of pulmonary congestion and the interventricular septal flattening (Fig. 2). Case 2: a middleaged man with liver cirrhosis was admitted for possible HRS type 1 after he presented with abdominal pain and found to have hyperbilirubinemia, elevated liver enzymes, and AKI. Renal function continued to worsen despite therapy with intravenous albumin, octreotide, and midodrine. Urine output dropped and diuretic therapy was contemplated. However, POCUS performed by the nephrology team revealed a small IVC, a predominantly A-line pattern on lung ultrasound with some B-lines at bases, and an estimated cardiac output of $9 \mathrm{~L}$ consistent with high-output state in the setting of splanchnic vaso- 
dilatation (Fig. 3). Renal resistive index was high at 0.83 , suggestive of hepatorenal pathophysiology. As these findings argue against both albumin (cardiac output is not low) and diuretic administration (no congestion), the patient was started on vasopressor therapy.

Another practical point that needs to be considered in this specific setting is that the Kidney Disease: Improving Global Outcomes (KDIGO) definition of AKI (i.e., serum creatinine increase $\geq 50 \%$ from baseline and/or $\geq 0.3 \mathrm{mg} /$ $\mathrm{dL}$ within $48 \mathrm{~h}$ ) that is commonly used by clinicians and investigators may not be applicable to patients with cirrhosis because of low creatinine production. In fact, it has been previously demonstrated that "normal" creatinine levels predict persistent kidney injury in these patients [15]. Furthermore, dilutional effect of intravascular volume expansion on serum creatinine is another confounding factor. This highlights the need for objective assessment of "congestive nephropathy" using Doppler ultrasonography and determination of "fluid tolerance" when intravenous fluid administration is contemplated [16].
Future studies should consider incorporating POCUSassisted physical examination prior to intravenous crystalloid or colloid therapy targeting a clinical or laboratory parameter to assess fluid tolerance and enhance patient safety.

\section{Conflict of Interest Statement}

The authors have no conflicts of interest to declare.

\section{Funding Sources}

The authors did not receive any funding.

\section{Author Contributions}

A.K. drafted the initial version of the manuscript and performed point-of-care ultrasound studies. C.R. and A.K. critically reviewed and revised the manuscript.

\section{References}

1 China L, Freemantle N, Forrest E, Kallis Y, Ryder SD, Wright G, et al. A randomized trial of albumin infusions in hospitalized patients with cirrhosis. N Engl J Med. 2021;384(9): 808-17.

2 Thévenot T, Bureau C, Oberti F, Anty R, Louvet $A$, Plessier A, et al. Effect of albumin in cirrhotic patients with infection other than spontaneous bacterial peritonitis. A randomized trial. J Hepatol. 2015;62(4):822-30.

3 Wong F, Pappas SC, Curry MP, Reddy KR, Rubin RA, Porayko MK, et al. Terlipressin plus albumin for the treatment of type 1 hepatorenal syndrome. N Engl J Med. 2021; 384(9):818-28.

4 Messmer AS, Zingg C, Müller M, Gerber JL, Schefold JC, Pfortmueller CA. Fluid overload and mortality in adult critical care patients-a systematic review and meta-analysis of observational studies. Crit Care Med. 2020;48(12): 1862-70.

5 RENAL Replacement Therapy Study Investigators; Bellomo R, Bellomo R, Cass A, Cole L, Finfer S, et al. An observational study fluid balance and patient outcomes in the randomized evaluation of normal vs. augmented level of replacement therapy trial. Crit Care Med. 2012;40(6):1753-60.
6 Cox EGM, Koster G, Baron A, Kaufmann T, Eck RJ, Veenstra TC, et al. Should the ultrasound probe replace your stethoscope? A SICS-I sub-study comparing lung ultrasound and pulmonary auscultation in the critically ill. Crit Care. 2020;24(1):14.

7 Velez JCQ, Petkovich B, Karakala N, Huggins JT. Point-of-care echocardiography unveils misclassification of acute kidney injury as hepatorenal syndrome. Am J Nephrol. 2019; 50(3):204-11.

8 Cotter G, Metra M, Milo-Cotter O, Dittrich HC, Gheorghiade M. Fluid overload in acute heart failure: re-distribution and other mechanisms beyond fluid accumulation. Eur J Heart Fail. 2008;10(2):165-9.

9 Kazory A, Ronco C. Hepatorenal syndrome or hepatocardiorenal syndrome: revisiting basic concepts in view of emerging data. Cardiorenal Med. 2019;9(1):1-7.

10 Koratala A, Kazory A. Point of care ultrasonography for objective assessment of heart failure: integration of cardiac, vascular, and extravascular determinants of volume status. Cardiorenal Med. 2021;11(1):5-17.

11 Wachsberg RH, Needleman L, Wilson DJ. Portal vein pulsatility in normal and cirrhotic adults without cardiac disease. J Clin Ultrasound. 1995;23(1):3-15.
12 Møller S, Bendtsen F. The pathophysiology of arterial vasodilatation and hyperdynamic circulation in cirrhosis. Liver Int. 2018 Apr; 38(4):570-80.

13 Umgelter A, Reindl W, Franzen M, Lenhardt C, Huber W, Schmid RM. Renal resistive index and renal function before and after paracentesis in patients with hepatorenal syndrome and tense ascites. Intensive Care Med. 2009;35(1):152-6.

14 Goyal S, Dixit VK, Jain AK, Shukla RC, Ghosh J, Kumar V. Intrarenal resistance index (RI) as a predictor of early renal impairment in patients with liver cirrhosis. Trop Gastroenterol. 2013;34(4):235-9.

15 Cullaro G, Park M, Lai JC. "Normal” creatinine levels predict persistent kidney injury and waitlist mortality in outpatients with cirrhosis. Hepatology. 2018;68(5):1953-60.

16 Beaubien-Souligny W, Rola P, Haycock K, Bouchard J, Lamarche Y, Spiegel R, et al. Quantifying systemic congestion with pointof-care ultrasound: development of the venous excess ultrasound grading system. Ultrasound J. 2020;12(1):16. 